\title{
Accelerate Monte Carlo Simulation for Probability Measures by an Interrupt Mechanism
}

\author{
Shuping Dang, Member, IEEE, Basem Shihada, Senior Member, IEEE, Mohamed-Slim Alouini, Fellow, IEEE
}

\begin{abstract}
Monte Carlo simulation (MCS) is useful for verifying analytical derivations and studying complex systems on an empirical basis. Although MCS is straightforward and does not require a priori knowledge of the sampled probability measures (PMs), it consumes a tremendous amount of computational resource and suffers from slow simulation procedures for sophisticated systems. To mitigate this drawback of MCS, we make full use of the monotone property and the logarithmic presentation convention of PMs in regard to power related metrics (PRMs) in communications science and propose an easy-to-implement interrupt mechanism to accelerate MCS for estimating PMs. To facilitate the programming on different platforms and provide a solid theoretical foundation, we present a generic implementation framework suited for estimating PMs with certain properties by MCS and analyze the underlying theory of the interrupt mechanism. In particular, we apply the de Moivre-Laplace theorem and analytic continuation to prove the asymptotic consistency under the indirect setup of MCS. We also design a hypothesis test for studying the proposed mechanism on a statistical basis.
\end{abstract}

Index Terms-Monte Carlo simulation, probability measure, interrupt mechanism, numerical computing, hypothesis test.

\section{INTRODUCTION}

$\mathbf{M}$ ONTE Carlo simulation (MCS) has been widely applied in almost all science and engineering subjects for numerical simulation and evaluation [1]. In communications science, MCS is commonly used to numerically estimating probability measures (PMs), e.g., outage probability and (bit/symbol/block) error probability [2], [3]. MCS is able to verify the correctness of analytical derivations and provide numerical insights into complex systems when mathematical analyses become intractable. Although MCS possesses a series of computing advantages, for example, the easy-to-implement, robust, and a priori knowledge free peculiarities, it inevitably demands a tremendous amount of time for processing enormous repeated random trials when approaching the statistical regularity in rare-event sampling by the law of large numbers [4]. This is in particular the case for complicated application scenarios in the context of sixth generation (6G) communications [5], e.g., reconfigurable intelligent surface (RIS) aided communication networks, artificial intelligence aided communication networks, space-air-ground integrated networks, and ultra-dense networks [6]-[10], and therefore researchers are in dire need of a fast MCS method.

Fortunately, most PMs studied in communications science for characterizing rare events, e.g., outage and error, are

The authors are with Computer, Electrical and Mathematical Science and Engineering Division, King Abdullah University of Science and Technology (KAUST), Thuwal 23955-6900, Saudi Arabia (e-mail: \{shuping.dang, basem.shihada, slim.alouini\}@ @aust.edu.sa). monotone non-increasing and presented in the logarithmic convention in regard to power related metrics (PRMs), including signal-to-interference-plus-noise ratio (SINR), signalto-noise ratio (SNR), and signal-to-interference ratio (SIR) [11]. We leverage these unique properties of PMs in communications science and propose an easy-to-implement interrupt mechanism in this letter to accelerate MCS for estimating PMs. In the rest of this letter, we give a generic implementation framework facilitating the implementations on different software platforms and analyze the underlying theory of the interrupt mechanism. By the mathematical analysis given in this letter, we provide an elementary proof of the asymptotic consistency of the estimates under the indirect setup of MCS that specifies a fixed total number of unfavorable events and counts the total number of repeated independent trials. Furthermore, a hypothesis test is also designed to rigorously study the interrupt mechanism and its statistical regularity.

Simulation results presented in this letter demonstrate that there exists a trade-off between estimation accuracy and simulation time when applying the interrupt mechanism, and the trade-off is controllable by several parameters. By properly setting these parameters, the simulation time yielded by the MCS with the interrupt mechanism can be reduced by an order of magnitude compared with that yielded by the naïve MCS without the interrupt mechanism, while the loss of estimation accuracy within the effective span of PRM is trivial.

It should be noted that the proposed method is dedicated to reduce the number of sampling rounds for the entire simulation procedure, instead of accelerating each round. Therefore, the proposed method is different from the widely applied sampling enhancement approaches for expediting each round, e.g., importance sampling, partial importance sampling, and probability-based sampling [12]-[14]. Instead, the proposed method can be applied in conjunction with these classical approaches in an overlaid manner and yields an additional performance gain.

\section{Rudiments of Probability Measures and Monte Carlo Simulation in Communications Science}

Strictly speaking, almost all performance measures of interest in communications science are probabilistic by nature. For the operational relevance, when talking about the PMs hereafter, we implicitly refer to the PMs with the following characteristics:

- A real-valued PM restricted within $[0,1]$ with a finite and binary support $\mathcal{S}$; 
- A PM capturing the statistical nature of a rare and unfavorable event ${ }^{1}$, and more specifically, evaluating the occurrence likelihood of such a random event;

- A PM that is monotone non-increasing with respect to PRMs, and by convention, presented in plots by a logarithmic coordinate with respect to a linear span of PRMs.

The outage probability and error probability in communications science are the representative examples of the PM with the aforementioned characteristics [11]. Without loss of generality, we denote such a symbolic PM as $P(\gamma)=\mathbb{P}\{$ unfavorable event occurs $\}$ with the support ${ }^{2} \mathcal{S}=$ \{unfavorable event, favorable event\}, where $\gamma$ represents a certain PRM expressed in decibel (dB) in general and can be reduced to either SINR, SIR, or SNR, depending on different contexts. By convention, $P(\gamma)$ is estimated by $\tilde{P}(\gamma)$ produced by MCS linearly with an equal step size spanning from the lowest $\gamma$ to the highest $\gamma$ of interest and presented in plots by a logarithmic coordinate [11].

Specifically, in most cases, $\tilde{P}(\gamma)$ is generated by MCS to estimate $P(\gamma)$ according to the following relation ${ }^{3}: \tilde{P}(\gamma)=$ $\mathrm{N}_{\mathrm{u}} / \tilde{\mathrm{N}}_{\text {tot }}(\gamma)$, where $\mathrm{N}_{\mathrm{u}}$ is a preset threshold of the collection of unfavorable events, and $\tilde{\mathrm{N}}_{\text {tot }}(\gamma)$ is the total number of repeated independent trials for collecting $\mathrm{N}_{\mathrm{u}}$ unfavorable events, which is random but statistically associated with $\gamma$. That is, we can repeatedly carry out a series of random and independent trials, check whether the unfavorable event occurs in each of these trials, and then terminate the MCS process once $N_{u}$ unfavorable events have been observed. In the meantime, we can count the total number of conducted trials $\tilde{\mathrm{N}}_{\text {tot }}(\gamma)$ when terminating the MCS process. With both figures, we are able to estimate $P(\gamma)$ by $\tilde{P}(\gamma)=\mathrm{N}_{\mathrm{u}} / \tilde{\mathrm{N}}_{\text {tot }}(\gamma)$. Because of the law of large numbers and statistical independence among all repeated trials, it can be proven that $\lim _{N_{u} \rightarrow \infty}\{\tilde{P}(\gamma)\}=P(\gamma)$ [15].

An illustration of a PM and its estimates generated by MCS with different $N_{u}$ in the logarithmic scale with respect to SNR $\gamma$ is presented in Fig. 1, which corresponds to the outage probability over a normalized Rayleigh fading environment. The numerical results presented in this figure give a straightforwardly visual impact of $\mathrm{N}_{\mathrm{u}}$ on the estimation accuracy.

\section{Monte Carlo Simulation with an Interrupt MECHANISM}

\section{A. Principle and Implementation Framework}

In communications science, researchers are normally interested in the outage/error performance above a certain threshold level $\eta$, say $10^{-2}$, which is prescribed by telecommunications standards and protocols. As a result, the performance below such a threshold is out of interest and might not be shown in plots. As an example shown in Fig. 1, setting $\eta=10^{-2}$

\footnotetext{
${ }^{1}$ Here, the rareness is a relative conception rather than a qualitative terminology. For example, in most communication systems functioning normally, both error and outage events should be rare.

${ }^{2}$ That is, either a favorable event, e.g., correct detection, or an unfavorable event, e.g., erroneous detection, will occur for each independent trial.

${ }^{3}$ This corresponds to the indirect setup of MCS, which is more efficient and robust than the direct setup specifying a fixed total number of repeated independent trials and counting a random number of unfavorable events.
}
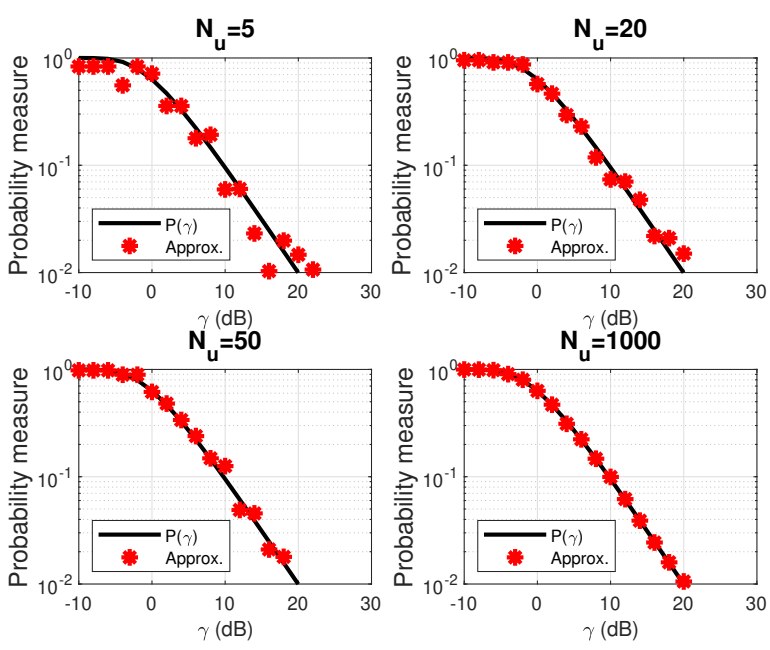

Fig. 1: Illustration of a PM and its estimates generated by MCS with different $\mathrm{N}_{\mathrm{u}}$ in the logarithmic scale with respect to $\gamma$.

as the threshold, the PM below this threshold has not been shown, although we sampled the PRM span up to $\gamma=30 \mathrm{~dB}$. That is, the sampling over the sub-span between $20 \mathrm{~dB}$ and 30 $\mathrm{dB}$ can be omitted. This naturally comes up with an idea that we can shrink the full span of $\gamma$ for sampling by terminating the simulation process at the value close to a critical PRM, denoted as $\gamma_{\mathrm{c}}$, which leads to $P\left(\gamma_{\mathrm{c}}\right)=\eta$. However, because of the lack of a priori knowledge ${ }^{4}$, when sampling over the full span of $\gamma$ with an equal step size, denoted as $\Theta$, we do not know the critical PRM $\gamma_{\mathrm{c}}$ beforehand.

Fortunately, given $\eta$, we can estimate $\gamma_{\mathrm{c}} \approx \gamma_{\mathrm{b}}=$ $\arg \min \left\{\left|\gamma-\gamma_{\mathrm{c}}\right|\right\} \in \Theta$ a posteriori by the numbers of $\gamma \in \Theta, \gamma>\gamma_{c}$

experienced trials and collected unfavorable events. Accordingly, we can stipulate an interrupt mechanism as follows based on a robust estimation of $\gamma_{c}$. In particular, when the number of counted conducted trials $\tilde{\mathrm{N}}_{\text {tot }}(\gamma)$ is equal to or larger than $\varepsilon \mathrm{N}_{\mathrm{u}} / \eta$, where $\varepsilon \geq 1$ is a control parameter balancing estimation accuracy and time, the MCS should be terminated. The corresponding $\gamma$ simulated at the termination point is estimated to be $\gamma_{\mathrm{b}}$. Therefore, we do not need to sample the PMs for the remaining span thereafter, which is equivalently to shrink $\Theta=\left[\min _{\Theta}\{\gamma\}, \ldots, \max _{\Theta}\{\gamma\}\right]$ to a sub-span $\Theta_{\mathrm{s}}=\left[\min _{\Theta}\{\gamma\}, \ldots, \gamma_{\mathrm{b}}\right]$. In this way, the overall simulation process can be accelerated.

To facilitate the implementation of the interrupt mechanism based acceleration for MCS, we design a generic implementation framework, regardless of the software platforms. Such a generic implementation framework is given by pseudocodes in Algorithm 1. As shown by the pseudocodes, the sampling is only carried out over a meaningful subset of $\gamma$ and will be terminated once the estimated PM is likely to be below threshold $\eta$ with a certain level of confidence controlled by $\varepsilon$.

\footnotetext{
${ }^{4}$ Even though sometimes we do have the a priori information, it is contradictory to the basic principle of MCS and should not be used for independent cross validation purposes.
} 


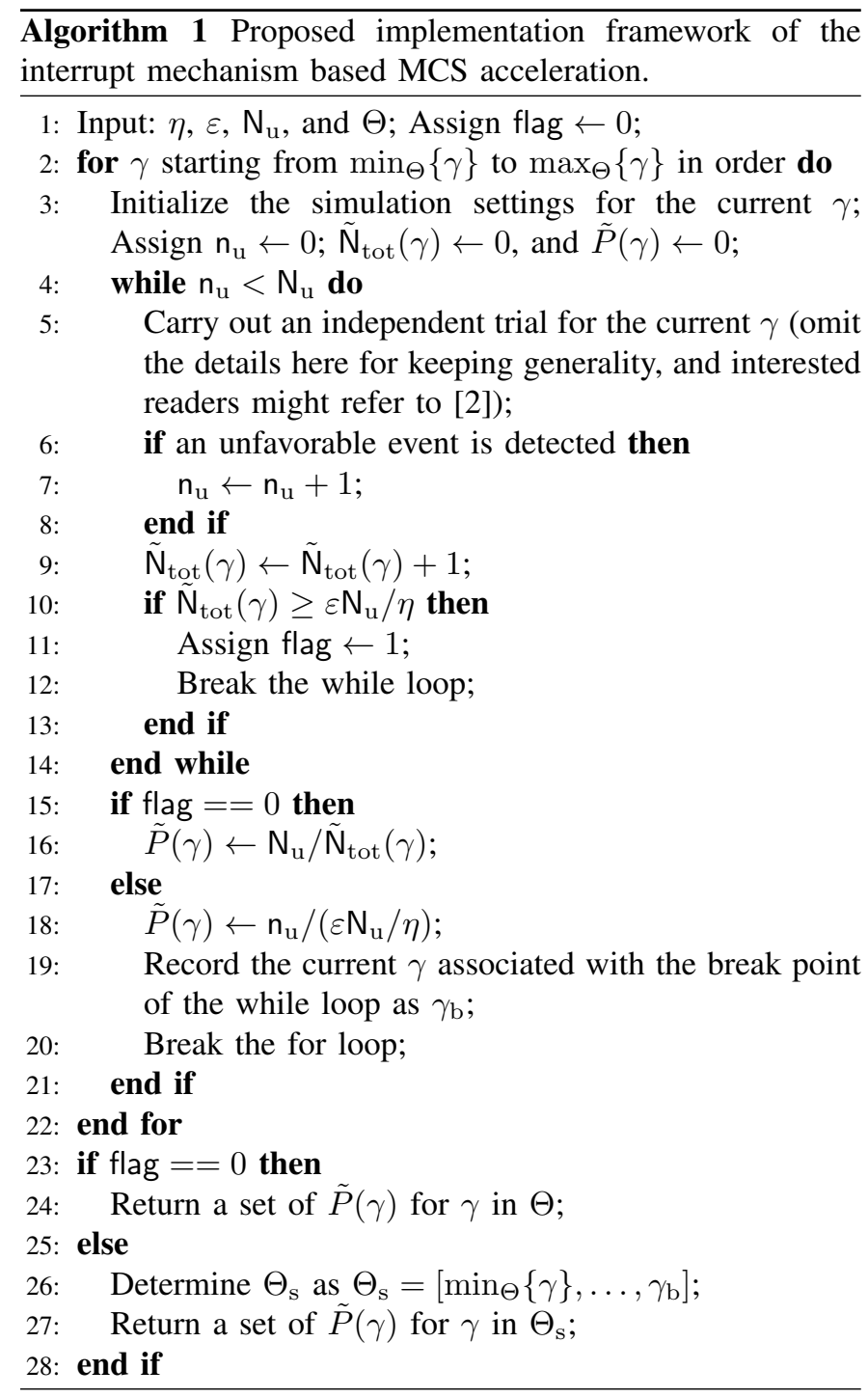

\section{B. Underlying Mathematical Theory}

To rigorously prove the effectiveness and efficiency of the proposed MCS approach enhanced by the interrupt mechanism, we reveal the underlying mathematical theory and analyze the statistical regularity in this subsection. The occurrence of a rare event can be modeled as a Bernoulli distribution (either occur or not) with the probability mass function only characterized by $P(\gamma)$, denoted as $\mathcal{B}(1, P(\gamma))$. Therefore, given a fixed number of collected rare events $\mathrm{N}_{\mathrm{u}}$, the number of experienced repeated independent trials $\tilde{N}_{\text {tot }}(\gamma)$ can be characterized by the following probability mass function (PMF): $\phi_{\tilde{N}_{\text {tot }}(\gamma)}(\alpha)=\mathbb{P}\left\{\tilde{\mathrm{N}}_{\text {tot }}(\gamma)=\alpha\right\}=$ $\left(\begin{array}{c}\alpha-1 \\ N_{\mathrm{u}-1}\end{array}\right)(P(\gamma))^{\mathrm{N}_{\mathrm{u}}}(1-P(\gamma))^{\alpha-\mathrm{N}_{\mathrm{u}}}$, where $\alpha \geq \mathrm{N}_{\mathrm{u}}$ is unbounded. By this PMF, the expectation and variance of $\tilde{N}_{\text {tot }}(\gamma)$ can be easily derived to be $\mathbb{E}\left\{\tilde{\mathrm{N}}_{\text {tot }}(\gamma)\right\}=\mathrm{N}_{\mathrm{u}} / P(\gamma)$ and $\mathbb{D}\left\{\tilde{\mathrm{N}}_{\text {tot }}(\gamma)\right\}=\mathrm{N}_{\mathrm{u}}(1-P(\gamma)) /(P(\gamma))^{2}$. Accordingly, we can derive the PMF of $\tilde{P}(\gamma)=\mathrm{N}_{\mathrm{u}} / \tilde{\mathrm{N}}_{\text {tot }}(\gamma)$ to be

$$
\begin{aligned}
\varrho_{\tilde{P}(\gamma)}(\beta) & =\mathbb{P}\{\tilde{P}(\gamma)=\beta\}=\mathbb{P}\left\{\tilde{\mathrm{N}}_{\text {tot }}(\gamma)=\mathrm{N}_{\mathrm{u}} / \beta\right\} \\
& =\left(\begin{array}{c}
\mathrm{N}_{\mathrm{u}} / \beta-1 \\
\mathrm{~N}_{\mathrm{u}}-1
\end{array}\right)(P(\gamma))^{\mathrm{N}_{\mathrm{u}}}(1-P(\gamma))^{\mathrm{N}_{\mathrm{u}} / \beta-\mathrm{N}_{\mathrm{u}}},
\end{aligned}
$$

where $\beta \in \mathbb{B}=\left\{\frac{N_{u}}{N_{u}+0}, \frac{N_{u}}{N_{u}+1}, \frac{N_{u}}{N_{u}+2}, \ldots\right\}$, which is a countably infinite set. The expectation of $\tilde{P}(\gamma)$ can also be determined as $\mathbb{E}\{\tilde{P}(\gamma)\}=P(\gamma){ }_{2} F_{1}\left(1,1 ; \mathrm{N}_{\mathrm{u}}+1 ; 1-P(\gamma)\right)$, where ${ }_{p} F_{q}(\cdot ; \cdot ; \cdot)$ is the generalized hypergeometric function. Because $\mathbb{E}\{\tilde{P}(\gamma)\} \neq P(\gamma), \tilde{P}(\gamma)$ is a biased estimate of $P(\gamma)$.

Meanwhile, we can prove the asymptotic consistency (a.k.a. the convergence in probability) of estimate $\tilde{P}(\gamma)$ by constructing $\lambda\left(\mathrm{N}_{\mathrm{u}}\right)=\mathbb{P}\{|\tilde{P}(\gamma)-P(\gamma)|<\epsilon\}=$ $\mathbb{P}\{P(\gamma)-\epsilon<\tilde{P}(\gamma)<P(\gamma)+\epsilon\} \geq \underline{\lambda}\left(\mathrm{N}_{\mathrm{u}}\right)=$ $\mathbb{P}\left\{z_{1} \leq \tilde{P}(\gamma) \leq z_{\mathrm{u}}\right\}=\sum_{\beta=z_{1}, \beta \in \mathbb{B}}^{z_{\mathrm{u}}}\left\{\varrho_{\tilde{P}(\gamma)}(\beta)\right\}$, where $\epsilon>0$ is an arbitrarily small value; $z_{1}$ and $z_{\mathrm{u}}$ are the lower and upper bounds on $\tilde{P}(\gamma)$ determined by $z_{1}=$ $\underset{z \in \mathbb{B}, z>P(\gamma)-\epsilon}{\arg \min }\{|z-(P(\gamma)-\epsilon)|\}=\frac{\mathrm{N}_{\mathrm{u}}}{\mathrm{N}_{\mathrm{u}}+\left\lfloor\frac{\mathrm{N}_{\mathrm{u}}}{P(\gamma)-\epsilon}-\mathrm{N}_{\mathrm{u}}\right\rfloor}$ and $z_{\mathrm{u}}=\underset{z \in \mathbb{B}, z<P(\gamma)+\epsilon}{\arg \min }\{|z-(P(\gamma)+\epsilon)|\}=\frac{\mathrm{N}_{\mathrm{u}}}{\mathrm{N}_{\mathrm{u}}+\left\lceil\frac{\mathrm{N}_{\mathrm{u}}}{P(\gamma)+\epsilon}-\mathrm{N}_{\mathrm{u}}\right\rceil}$, where $\lfloor\cdot\rfloor$ and $\lceil\cdot\rceil$ represent the floor and ceiling functions, returning the nearest integers in the directions of negative and positive infinity, respectively.

However, when $\mathrm{N}_{\mathrm{u}}$ is large, it is cumbersome to analyze $\varrho_{\tilde{P}(\gamma)}(\beta)$ because of the involvement of the binomial coefficient. To facilitate the following analysis, we apply the following approximation derived from the de Moivre-Laplace theorem for large $\mathrm{N}_{\mathrm{u}}[15]$ :

$$
\varrho_{\tilde{P}(\gamma)}(\beta) \approx \hat{\varrho}_{\tilde{P}(\gamma)}(\beta)=\frac{P(\gamma) \exp \left(-\frac{\left[N_{\mathrm{u}}-1-\left(\frac{N_{\mathrm{u}}}{\beta}-1\right) P(\gamma)\right]^{2}}{2\left(\frac{N_{\mathrm{u}}}{\beta}-1\right) P(\gamma)(1-P(\gamma))}\right)}{\sqrt{2 \pi\left(\frac{\mathrm{N}_{\mathrm{u}}}{\beta}-1\right) P(\gamma)(1-P(\gamma))}} .
$$

That is, because the interval between two consecutive values in $\mathbb{B}$, i.e., $\Delta(\beta)=\beta^{2} /\left(\mathrm{N}_{\mathrm{u}}-\beta\right)$, will become infinitesimal when $\mathrm{N}_{\mathrm{u}} \rightarrow \infty$, we can utilize a continuous distribution, a.k.a. the limiting distribution, characterized by probability density function (PDF) $\varrho_{\tilde{P}(\gamma)}(\beta) / \Delta(\beta)$ to approximate the original discrete distribution characterized by $\operatorname{PMF} \varrho_{\tilde{P}(\gamma)}(\beta)$. Accordingly, we apply the techniques of analytic continuation and extend the domain of definition for $\tilde{P}(\gamma)$ from $\mathbb{B}$ to $[0,1]$. Consequently, we can approximate the cumulative distribution function (CDF) of $\tilde{P}(\gamma)$ for large $\mathrm{N}_{\mathrm{u}}$ by

$$
\begin{aligned}
& \mathbb{P}\{\tilde{P}(\gamma) \leq \beta\} \approx \hat{\Xi}_{\tilde{P}(\gamma)}(\beta)=\int_{0}^{\beta} \hat{\varrho}_{\tilde{P}(\gamma)}(t) / \Delta(t) \mathrm{d} t \\
& \approx \frac{1}{2} \operatorname{erfc}\left(\frac{\sqrt{\frac{\mathrm{N}_{\mathrm{u}} P(\gamma)}{1-P(\gamma)}}-\beta \sqrt{\frac{\mathrm{N}_{\mathrm{u}}}{P(\gamma)(1-P(\gamma))}}}{\sqrt{2 \beta}}\right) \\
& +\frac{1}{2} \exp \left(\frac{2 \mathrm{~N}_{\mathrm{u}}}{1-P(\gamma)}\right) \operatorname{erfc}\left(\frac{\sqrt{\frac{\mathrm{N}_{\mathrm{u}} P(\gamma)}{1-P(\gamma)}}+\beta \sqrt{\frac{\mathrm{N}_{\mathrm{u}}}{P(\gamma)(1-P(\gamma))}}}{\sqrt{2 \beta}}\right),
\end{aligned}
$$

where $\operatorname{erfc}(\cdot)$ is the complementary error function. This approximate result leads to $\underline{\lambda}\left(\mathrm{N}_{\mathrm{u}}\right) \approx \int_{z_{1}}^{z_{\mathrm{u}}} \hat{\varrho}_{\tilde{P}(\gamma)}(t) / \Delta(t) \mathrm{d} t=$ $\hat{\Xi}_{\tilde{P}(\gamma)}\left(z_{\mathrm{u}}\right)-\hat{\Xi}_{\tilde{P}(\gamma)}\left(z_{1}\right)$ for large $\mathrm{N}_{\mathrm{u}}$. It can be calculated that $\lim _{\mathrm{N}_{\mathrm{u}} \rightarrow \infty}\left\{\underline{\lambda}\left(\mathrm{N}_{\mathrm{u}}\right)\right\} \approx \lim _{\mathrm{N}_{\mathrm{u}} \rightarrow \infty}\left\{\int_{z_{1}}^{z_{\mathrm{u}}} \hat{\varrho}_{\tilde{P}(\gamma)}(t) / \Delta(t) \mathrm{d} t\right\}=1$, and, by the squeeze theorem, because $1 \geq$ $\lim _{N_{\mathrm{u}} \rightarrow \infty}\left\{\lambda\left(\mathrm{N}_{\mathrm{u}}\right)\right\} \geq \lim _{\mathrm{N}_{\mathrm{u}} \rightarrow \infty}\left\{\underline{\lambda}\left(\mathrm{N}_{\mathrm{u}}\right)\right\} \approx 1$, we have 


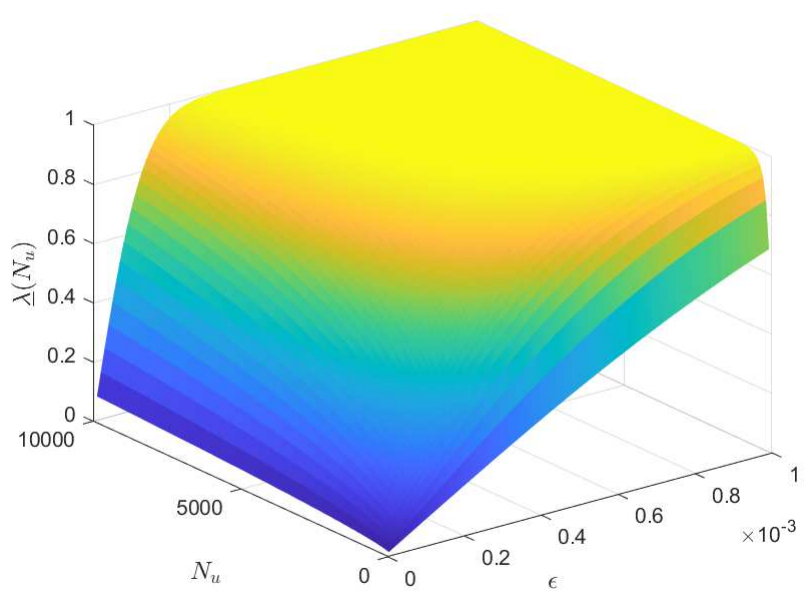

Fig. 2: Relation among $\underline{\lambda}\left(\mathrm{N}_{\mathrm{u}}\right), \mathrm{N}_{\mathrm{u}}$, and $\epsilon$, given $P(\gamma)=10^{-2}$.

$\lim _{N_{u} \rightarrow \infty}\left\{\lambda\left(N_{u}\right)\right\}=1$. Therefore, the asymptotic consistency has been proven by $\forall \epsilon>0, \exists \lim _{N_{u} \rightarrow \infty}\left\{\lambda\left(N_{u}\right)\right\}=1$, leading to $\tilde{P}(\gamma) \stackrel{\mathrm{P}}{\longrightarrow} P(\gamma)$. To verify this verdict, we plot $\underline{\lambda}\left(\mathrm{N}_{\mathrm{u}}\right)$ represented by its continuous approximation versus $\mathrm{N}_{\mathrm{u}}$ and $\epsilon$, given $P(\gamma)=10^{-2}$, in Fig. 2. As shown in this figure, $\forall \epsilon>0$, we can always have a large value of $\mathrm{N}_{\mathrm{u}}$ ensuring that $\underline{\lambda}\left(\mathrm{N}_{\mathrm{u}}\right)$ is sufficiently close to 1 .

\section{Hypothesis Test Design}

To rigorously investigate the nature of the proposed interrupt mechanism and quantify its effectiveness, we design the following hypothesis test when the interrupt mechanism is triggered, given $\tilde{\mathrm{N}}_{\text {tot }}(\gamma)=\varepsilon \mathrm{N}_{\mathrm{u}} / \eta$ and the number of observed unfavorable events $n_{u} \leq N_{u}$ :

$$
H_{0}: P(\gamma) \leq \eta, \quad H_{1}: P(\gamma)>\eta
$$

To facilitate the analysis of the hypothesis test, we introduce an auxiliary Bernoulli distributed random variable indicating whether an unfavorable event independently occurs in the $n$th trial or not: $A_{n} \sim \mathcal{B}(1, P(\gamma))$. Then, for simplicity, we approximate $\mathrm{n}_{\mathrm{u}}$ as $\mathrm{n}_{\mathrm{u}} \approx \sum_{n=1}^{\varepsilon \mathrm{N}_{\mathrm{u}} / \eta} A_{n}$. Now, with large $\mathrm{N}_{\mathrm{u}}$, we can apply the Lyapunov theorem and approximately have

$$
\mathrm{n}_{\mathrm{u}} /\left(\varepsilon \mathrm{N}_{\mathrm{u}} / \eta\right) \sim \mathcal{N}\left(P(\gamma), \frac{P(\gamma)(1-P(\gamma))}{\varepsilon \mathrm{N}_{\mathrm{u}} / \eta}\right) .
$$

By this approximation, the statistic analysis can be greatly facilitated, and the hypothesis test formulated in (4) becomes a hypothesis test for the expectation of a Gaussian distributed random variable. Therefore, by normalizing the Gaussian random variable, we can construct a test statistic

$$
\mathrm{T}=\left(\frac{\mathrm{n}_{\mathrm{u}}}{\varepsilon \mathrm{N}_{\mathrm{u}}}-1\right) \sqrt{\frac{\varepsilon \mathrm{N}_{\mathrm{u}}}{1-\eta}} \sim \mathcal{N}(0,1),
$$

which gives the approximate region of rejection for the hypothesis formulated test in (4) to be $T \geq u(\omega)$ [15], where $\omega$ is the level of statistical significance, and $u(\omega)$ is the upper quantile function for the standard Gaussian distribution $\mathcal{N}(0,1)$ determined by $\int_{u(\omega)}^{\infty} \exp \left(-t^{2} / 2\right) /(\sqrt{2 \pi}) \mathrm{d} t=\omega$.
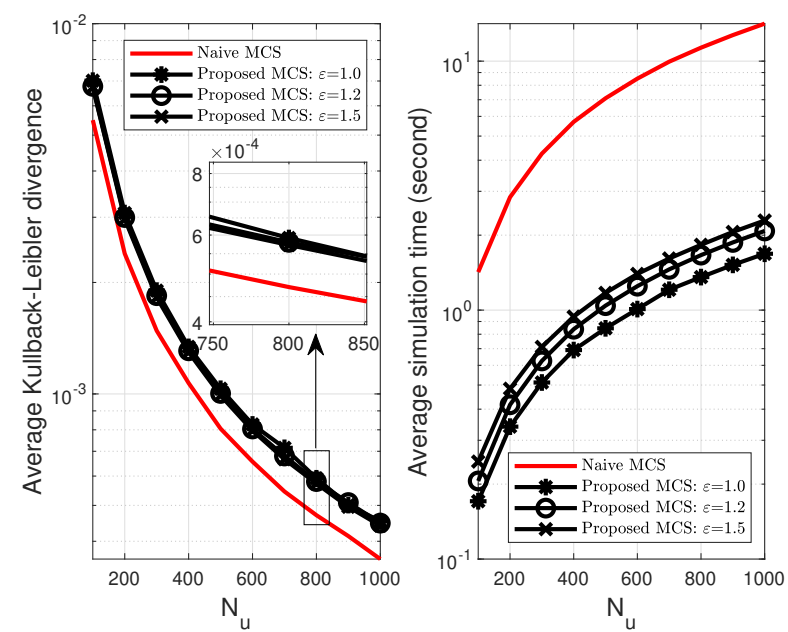

Fig. 3: Experimental results of average Kullback-Leibler divergence and simulation time vs. the number of observed outage events $N_{u}$.

\section{EXPERIMENTAL RESUlTS AND DisCUSSION}

In general, the performance of MCS is characterized by the estimation accuracy, measuring how close the numerical results are to the analytical results, and the simulation time, measuring how long the simulation process takes. Although it is easy to identify the estimation accuracy from a qualitative perspective from Fig. 1, it is relatively cumbersome to quantify the estimation accuracy by MCS. Classical studies resort to the goodness-of-fit for the quantifying purposes, while it has been shown to be inadequate for rare-event PM that is in general a very small value. In this letter, we utilize the Kullback-Leibler divergence (a.k.a. relative entropy) as the estimation accuracy measure to get rid of the effect of the small value of PM per se [16]. The occurrence of a favorable or unfavorable event is essentially Bernoulli distributed, and thus the distance measure between $P(\gamma)$ and $\tilde{P}(\gamma)$ can be explicitly written as

$\operatorname{KLD}(\gamma)=\tilde{P}(\gamma) \log _{2}\left(\frac{\tilde{P}(\gamma)}{P(\gamma)}\right)+(1-\tilde{P}(\gamma)) \log _{2}\left(\frac{1-\tilde{P}(\gamma)}{1-P(\gamma)}\right)$

To take the interrupt mechanism into consideration, we can further average $\operatorname{KLD}(\gamma)$ over the effective span $\Theta_{\mathrm{s}}$ of $\gamma$ to obtain

$$
\overline{\mathrm{KLD}}=\underset{\gamma \in \Theta_{\mathrm{s}}}{\mathbb{E}}\{\mathrm{KLD}(\gamma)\}=\frac{1}{\left|\Theta_{\mathrm{s}}\right|} \sum_{\gamma \in \Theta_{\mathrm{s}}} \operatorname{KLD}(\gamma)
$$

Apart from the estimation accuracy, simulation time Time $(\gamma)$ is another crucial indicator of the performance of the proposed method, which signifies the computational complexity in a practical context. We set up the simulation environment with the following specifications: Processor: $\operatorname{Intel}(\mathrm{R}) \mathrm{Xeon}(\mathrm{R})$ Gold $6130 \mathrm{CPU} @ 2.10 \mathrm{GHz}-2.10 \mathrm{GHz}$ (two processors); installed memory (RAM): 128 GB; operating system: 64bit Windows 10, and count the simulation time $\operatorname{Time}(\gamma)$ by tic/toc function embedded on MATLAB R2018a.

To reveal the computing generality, we adopt the outage probability over a normalized Rayleigh fading environment with SNR $\gamma \in\{-10,-9, \ldots, 30\}(\mathrm{dB})$ and $\eta=10^{-2}$ as 
a simplistic example to investigate. For such a simplistic case, it can be mathematically derived that $\gamma_{\mathrm{c}}=-(\log (1-$ $\eta))^{-1} \approx 19.9782 \mathrm{~dB}$ for the normalized channel setup [3]. The experimental results regarding the average Kullback-Leibler divergence and simulation time are presented in Fig. 3. To reveal the statistical regularity, we repeat the experiments for $10^{4}$ times and average the results over all runs.

The naïve MCS method is taken as the comparison benchmark in these measurements. From Fig. 3, it can be verified that the estimation accuracy loss caused by the interrupt mechanism is acceptable over the entire region of $\mathrm{N}_{\mathrm{u}}$. In fact, the procedures of naïve MCS and the proposed MCS are the same before reaching $\gamma_{\mathrm{b}}$, and the difference is only caused by the incomplete simulation procedure for the remaining span after $\gamma_{\mathrm{b}}$. On the other hand, as also shown in Fig. 3, with such an interrupt mechanism, the simulation time corresponding to the proposed MCS method has been significantly reduced, since the simulation procedure can be terminated earlier. Based on the above observations, the effectiveness and efficiency of the MCS enhanced by the interrupt mechanism have been validated. Furthermore, letting $\omega=0.05$, most test statistics $\mathrm{T}$ of the above experiments are smaller than $u(\omega)$ when the interrupt mechanism is triggered. Therefore, the null hypothesis in (4) cannot be rejected, which supports that the PMs are smaller than threshold $\eta$ in all experiments when the interrupt mechanism is triggered.

\section{CONCLusion}

To meet the ever-increasing demands on fast simulations for complex communication systems, we proposed an easyto-implement interrupt mechanism in this letter to accelerate the MCS procedure for PMs with certain characteristics. To be general and rigorous, we also presented a generic implementation framework described in pseudocodes and analyzed its underlying mathematical theory. As validated by experimental results and hypothesis tests, the MCS method enhanced by the interrupt mechanism is capable of achieving a similar level of estimation accuracy compared to the benchmark but requires much less simulation time. It is anticipated that the methods presented in this letter will help to expedite the research progress of complex $6 \mathrm{G}$ communication systems.

\section{REFERENCES}

[1] A. Shapiro, "Monte Carlo sampling methods," Handbooks in operations research and management science, vol. 10, pp. 353-425, 2003.

[2] J. Proakis, M. Salehi, and G. Bauch, Contemporary Communication Systems Using MATLAB. Cengage Learning, 2012.

[3] Z. Wang, S. Dang, S. Shaham, Z. Zhang, and Z. Lv, "Basic research methodology in wireless communications: The first course for researchbased graduate students," IEEE Access, vol. 7, pp. 86678-86 696, 2019.

[4] M.-H. Hsieh, "Adaptive Monte Carlo methods for rare event simulations," in Proc. IEEE WSC, vol. 1, San Diego, USA, 2002, pp. 108-115.

[5] S. Dang, O. Amin, B. Shihada, and M.-S. Alouini, "What should 6G be?" Nature Electronics, vol. 3, no. 1, pp. 20-29, 2020.

[6] E. Basar, "Reconfigurable intelligent surface-based index modulation: A new beyond MIMO paradigm for 6G," IEEE Trans. Commun., vol. 68, no. 5, pp. 3187-3196, 2020.

[7] M. Chen, U. Challita, W. Saad, C. Yin, and M. Debbah, "Artificial neural networks-based machine learning for wireless networks: A tutorial," IEEE Commun. Surv. Tutor., vol. 21, no. 4, pp. 3039-3071, 2019.
[8] J. Kang, Z. Xiong, D. Niyato, Y. Zou, Y. Zhang, and M. Guizani, "Reliable federated learning for mobile networks," IEEE Wireless Communications, vol. 27, no. 2, pp. 72-80, 2020.

[9] J. Ye, S. Dang, B. Shihada, and M.-S. Alouini, "Space-air-ground integrated network: Outage performance analysis," IEEE Trans. Wirel. Commun., 2020 (early access article).

[10] Y. Chen, M. Ding, and D. López-Pérez, "Performance of ultra-dense networks with a generalized multipath fading," IEEE Wirel. Commun. Lett., vol. 8, no. 5, pp. 1419-1422, 2019.

[11] Z. Wang and G. B. Giannakis, "A simple and general parameterization quantifying performance in fading channels," IEEE Trans. Commun., vol. 51, no. 8, pp. 1389-1398, 2003.

[12] N. Ben Rached, A. Kammoun, M.-S. Alouini, and R. Tempone, "Unified importance sampling schemes for efficient simulation of outage capacity over generalized fading channels," IEEE J. Sel. Top. Signal Process., vol. 10, no. 2, pp. 376-388, 2016.

[13] R. Yuan, J. Ma, P. Su, Y. Dong, and J. Cheng, "Monte-Carlo integration models for multiple scattering based optical wireless communication," IEEE Transactions on Communications, vol. 68, no. 1, pp. 334-348, 2020.

[14] D. K. Borah, V. R. Mareddy, and D. G. Voelz, "Single and double scattering event analysis for ultraviolet communication channels," Optics Express, vol. 29, no. 4, pp. 5327-5342, 2021.

[15] A. Papoulis and S. Pillai, Probability, Random Variables, and Stochastic Processes, ser. McGraw-Hill series in electrical engineering: Communications and signal processing. McGraw-Hill, 2002.

[16] J. Watson, L. Nieto-Barajas, and C. Holmes, "Characterizing variation of nonparametric random probability measures using the Kullback-Leibler divergence," Statistics, vol. 51, no. 3, pp. 558-571, 2017. 